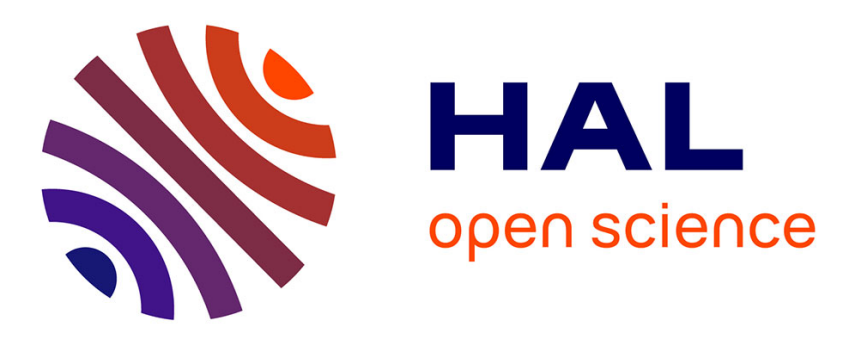

\title{
A nonlinear Korn inequality with boundary conditions and its relation to the existence of minimizers in nonlinear elasticity
}

\author{
Cristinel Mardare
}

\section{- To cite this version:}

Cristinel Mardare. A nonlinear Korn inequality with boundary conditions and its relation to the existence of minimizers in nonlinear elasticity. Comptes rendus de l'Académie des sciences. Série I, Mathématique, 2011, 349, pp.229 - 232. 10.1016/j.crma.2011.01.011 . hal-01077300

\section{HAL Id: hal-01077300 \\ https: / hal.sorbonne-universite.fr/hal-01077300}

Submitted on 24 Oct 2014

HAL is a multi-disciplinary open access archive for the deposit and dissemination of scientific research documents, whether they are published or not. The documents may come from teaching and research institutions in France or abroad, or from public or private research centers.
L'archive ouverte pluridisciplinaire HAL, est destinée au dépôt et à la diffusion de documents scientifiques de niveau recherche, publiés ou non, émanant des établissements d'enseignement et de recherche français ou étrangers, des laboratoires publics ou privés. 


\title{
A nonlinear Korn inequality with boundary conditions and its relation to the existence of minimizers in nonlinear elasticity
}

\author{
Cristinel Mardare \\ Université Pierre et Marie Curie - Paris 6, Laboratoire Jacques - Louis Lions, Paris, F-75005 France \\ Received November 2010 \\ Presented by Philippe G. Ciarlet
}

\begin{abstract}
We establish a nonlinear Korn inequality with boundary conditions showing that the $H^{1}$-distance between two mappings from $\Omega \subset \mathbb{R}^{n}$ into $\mathbb{R}^{n}$ preserving orientation is bounded, up to a multiplicative constant, by the $L^{2}$ distance between their metrics. This inequality is then used to show the existence of a unique minimizer to the total energy of a hyperelastic body, under the assumptions that the $L^{p}$-norm of the density of the applied forces is small enough and the stored energy function is bounded from below by a positive definite quadratic function of the Green - Saint Venant strain tensor. To cite this article: C. Mardare, C. R. Acad. Sci. Paris, Ser. I 34x (201x).

Résumé

Une inégalité de Korn non linéaire et son relation à l'existence de minimiseurs en elasticité non linéaire Nous établissons une inégalité de Korn non linéaire avec conditions au bord montrant que la distance dans $H^{1}$ entre deux applications de $\Omega \subset \mathbb{R}^{n}$ à $\mathbb{R}^{n}$ préservant l'orientation est majorée, à une constante multiplicative près, par la distance dans $L^{2}$ entre leurs métriques. Cette inégalité est ensuite utilisée pour montrer l'existence d'un minimiseur unique de l'énergie totale d'un corps hyperélastique, sous les hypothèses que la norme de la densité des forces appliquées est suffisamment petite en norme $L^{p}$, et la densité d'énergie de déformation est minorée par une fonction quadratique du tenseur de Green - Saint Venant. Pour citer cet article : C. Mardare, C. R. Acad. Sci. Paris, Ser. I $34 x$ (201x).
\end{abstract}

\section{Introduction and main results}

We use the following notation. $\mathbb{M}^{n}, \mathbb{S}^{n}, \mathbb{M}_{+}^{n}$, and $\mathbb{O}_{+}^{n}$ denote respectively the space of square matrices of order $n$, the space of all symmetric matrices of order $n$, the set of all matrices $A \in \mathbb{M}^{n}$ such that $\operatorname{det} A>0$, and the set of all matrices $A \in \mathbb{M}_{+}^{n}$ such that $A^{T} A=I . A^{T}$ and $I$ denote respectively the transpose of the matrix $A$ and the identity matrix in $\mathbb{M}^{n}$. The inner products in $\mathbb{R}^{n}$ and $\mathbb{M}^{n}$ are respectively denoted - and : . The Euclidean norm in $\mathbb{R}^{n}$ and the Frobenius norm in $\mathbb{M}^{n}$ are both denoted by $|\cdot|$. Vector and matrix fields are denoted by boldface letters. The gradient of a vector field $\mathbf{u}$ is the matrix field $\nabla \mathbf{u}$, whose columns are the partial derivatives of $\mathbf{u}$. The divergence of a matrix field $\mathbf{A}$ is the vector field $\operatorname{div} \mathbf{A}$, whose components are the divergences of the row vectors of $\mathbf{A}$. The matrix field $\mathbf{I}$ is defined by $\mathbf{I}(x)=I$ for all $x \in \mathbb{R}^{n} . L^{p}(\Omega), W^{k, p}(\Omega)$ or $H^{k}(\Omega)$ if $p=2$, and $\mathcal{C}^{k}(\Omega)$ denote respectively Lebesgue spaces, Sobolev spaces, and $k$ times continuously differentiable functions.

The first result of this Note is the following nonlinear Korn inequality with boundary conditions:

Theorem 1 Let $\Omega$ be a bounded connected open subset of $\mathbb{R}^{n}$ with a Lipschitz-continuous boundary $\partial \Omega$ and let $\lambda<1$. Then there exists a constant $C$ such that

$$
\|\nabla \mathbf{u}-\nabla \mathbf{v}\|_{L^{2}(\Omega)} \leq \frac{C}{(1-\lambda)^{2+n / 2}}\left\|\nabla \mathbf{u}^{T} \nabla \mathbf{u}-\nabla \mathbf{v}^{T} \nabla \mathbf{v}\right\|_{L^{2}(\Omega)}
$$

for all pairs $(\mathbf{u}, \mathbf{v}) \in W^{1,4}\left(\Omega ; \mathbb{R}^{n}\right) \times \mathcal{C}^{1}\left(\bar{\Omega} ; \mathbb{R}^{n}\right)$ that satisfy $\mathbf{u}(x)=\mathbf{v}(x)$ for almost all $x \in \partial \Omega$, $\operatorname{det}(\nabla \mathbf{u}(x))>$ 0 for almost all $x \in \Omega, \mathbf{v}$ is injective, and $\|\nabla \mathbf{v}-\mathbf{I}\|_{L^{\infty}(\Omega)} \leq \lambda$.

Email address: mardare@ann.jussieu.fr (Cristinel Mardare). 
Note that the above inequality remains valid for deformation fields that are possibly non injective and satisfy more general boundary conditions, as will be shown elsewhere.

The inequality of Theorem 1 is reminiscent of another inequality, established earlier by Ciarlet \& Mardare [4], showing that, given any vector field $\mathbf{v} \in \mathcal{C}^{1}\left(\bar{\Omega} ; \mathbb{R}^{n}\right)$ satisfying $\operatorname{det} \nabla \mathbf{v}>0$ in $\bar{\Omega}$, there exists a constant $C(\mathbf{v})$ such that

$$
\inf _{R \in \mathbb{O}_{+}^{n}}\|\nabla \mathbf{u}-R \nabla \mathbf{v}\|_{L^{2}(\Omega)} \leq C(\mathbf{v})\left\|\nabla \mathbf{u}^{T} \nabla \mathbf{u}-\nabla \mathbf{v}^{T} \nabla \mathbf{v}\right\|_{L^{1}(\Omega)}^{1 / 2}
$$

for all $\mathbf{u} \in H^{1}\left(\Omega ; \mathbb{R}^{n}\right)$ satisfying $\operatorname{det} \nabla \mathbf{u}>0$ a.e. in $\Omega$. The idea that the exponent $1 / 2$ can be dropped at the expense of a stronger norm in the right-hand side of the last inequality is due to Blanchard [2].

Theorem 1 has applications in nonlinear elasticity. Consider a body with reference configuration $\Omega \subset$ $\mathbb{R}^{3}$, made of a hyperelastic material characterized by a stored energy function $\hat{W}: \Omega \times \mathbb{M}_{+}^{3} \rightarrow \mathbb{R}$, and subjected to dead body forces of density $\mathbf{f}: \Omega \rightarrow \mathbb{R}^{3}$. Then the total energy corresponding to a deformation $\mathbf{u}: \Omega \rightarrow \mathbb{R}^{3}$ of the body is given by

$$
J(\mathbf{u})=\int_{\Omega} \hat{W}(x, \nabla \mathbf{u}(x)) d x-\int_{\Omega} \mathbf{f}(x) \cdot \mathbf{u}(x) d x .
$$

If $\hat{W}$ is polyconvex and satisfies suitably growth conditions, then $J$ possesses a minimizer over a suitable set of admissible deformations $\mathbf{u}$; cf. Ball [1]. If in addition the Euler-Lagrange equation corresponding to this minimization problem has a solution by the implicit function theorem, then this solution coincides with the minimizer above; cf. Zhang [8].

The second result of this Note is that the total energy $J$ possesses a minimizer over a suitable set of admissible deformations $\mathbf{u}$ for some hyperelastic materials that does not necessarily meet the assumptions of Ball and Zhang. We assume that the stored energy function $\hat{W}$ satisfies the axiom of material frameindifference, so that there exists a function $W: \Omega \times \mathbb{S}^{3} \rightarrow \mathbb{R}$ such that

$$
\hat{W}(x, F)=W(x, E), E=\frac{1}{2}\left(F^{T} F-I\right) \text { for almost all } x \in \Omega \text { and for all } F \in \mathbb{M}_{+}^{3} .
$$

We recall that $\Omega$ is a natural configuration of the body if $\frac{\partial W}{\partial E}(x, 0)=0$ for all $x \in \Omega$.

Theorem 2 Let $\Omega$ be a bounded connected open subset of $\mathbb{R}^{3}$ with a boundary of class $\mathcal{C}^{2}$ and let $\mathbf{f} \in$ $L^{p}\left(\Omega ; \mathbb{R}^{3}\right), p>3$. Assume that $W \in \mathcal{C}^{3}\left(\bar{\Omega} \times \mathbb{S}^{3}\right)$, that $\frac{\partial W}{\partial E}(x, 0)=0$ for all $x \in \Omega$, and that there exist constants $\alpha>0$ and $\varepsilon>0$ such that

$$
W(x, E+H) \geq W(x, E)+\frac{\partial W}{\partial E}(x, E): H+\alpha|H|^{2} \quad \text { for all } x \in \bar{\Omega}, H \in \mathbb{S}^{3}, E \in \mathbb{S}^{3},|E|<\varepsilon .
$$

Then there exists a constant $\delta>0$ with the following property: If $\|\mathbf{f}\|_{L^{p}(\Omega)}<\delta$, then the functional $J$ defined by the equations (1)-(2) has a unique minimizer over the set of admissible deformations defined by

$$
\mathcal{M}=\left\{\mathbf{u} \in W^{1,4}\left(\Omega ; \mathbb{R}^{3}\right) ; \operatorname{det}(\nabla \mathbf{u})>0 \text { a.e. in } \Omega, \mathbf{u}(x)=x \text { for all } x \in \partial \Omega\right\} .
$$

Moreover, the minimizer $\mathbf{v}$ belongs to the space $\mathcal{C}^{1}\left(\bar{\Omega} ; \mathbb{R}^{3}\right)$, is injective from $\bar{\Omega}$ into $\mathbb{R}^{3}$, and satisfies $\operatorname{det} \nabla \mathbf{v}(x)>0$ for all $x \in \bar{\Omega}$.

Theorem 2 applies in particular to the stored energy function of a Saint Venant - Kirchhoff material, given in terms of its Lamé constants $\lambda>0$ and $\mu>0$ by the expression

$$
\hat{W}(x, F)=W(x, E)=\frac{\lambda}{2}(\operatorname{tr} E)^{2}+\mu|E|^{2}, E=\frac{1}{2}\left(F^{T} F-I\right), \text { for all }(x, F) \in \Omega \times \mathbb{M}_{+}^{3} .
$$

Note that the previous existence theorems of Ball [1], Zhang [8], and Ciarlet \& Mardare [5] do not apply in that case.

Theorem 2 still holds if $W$ is of class $\mathcal{C}^{3}$ only over the set $\bar{\Omega} \times\left\{E \in \mathbb{S}^{3} ;|E|<\varepsilon\right\}$ for some $\varepsilon>0$. Note also that the assumptions of Theorem 2 imply that the mapping $E \mapsto W(x, E)$ is convex in a neighborhood of the zero matrix. But this does not imply that the mapping $F \mapsto \hat{W}(x, F)$ is convex in a neighborhood of the identity matrix ( $\hat{W}$ and $W$ are related by $(2)$ ), so the direct methods in the calculus of variations cannot be used to prove the existence of a minimizer of the functional $J$ defined by (1). 


\section{Proof of Theorem 1}

Extend the mapping $\mathbf{v}$ to an open ball $B \subset \mathbb{R}^{n}$ containing $\bar{\Omega}$ and let $\mathbf{u}(x)=\mathbf{v}(x)$ for all $x \in B \backslash \bar{\Omega}$. Define the composite mapping $\varphi:=\mathbf{u} \circ \mathbf{v}^{-1}$ and note that $\varphi \in W^{1,4}\left(B ; \mathbb{R}^{n}\right)$ and $\operatorname{det}(\nabla \varphi(x))>0$ for almost all $x \in \bar{B}$; cf. Ciarlet [3, Theorems 5.5.1 and 5.5-2].

The geometric rigidity lemma of Friesecke, James \& Müller [6, Theorem 3.1] implies that there exists a constant $K$ independent of $\mathbf{u}$ and $\mathbf{v}$ such that

$$
\inf _{R \in \mathbb{O}_{+}^{n}} \int_{B}|\nabla \varphi(x)-R|^{2} d x \leq K \int_{B} \inf _{Q \in \mathbb{O}_{+}^{n}}|\nabla \varphi(x)-Q|^{2} d x .
$$

The identity $I=\frac{1}{|B \backslash \Omega|} \int_{B \backslash \Omega} \nabla \varphi(x) d x$, where $|B \backslash \Omega|$ denotes the Lebesque measure of the set $B \backslash \Omega$, implies that

$$
\|\nabla \varphi-\mathbf{I}\|_{L^{2}(B)} \leq\left(\int_{B}|\nabla \varphi(x)-R|^{2} d x\right)^{1 / 2}+\frac{|B|^{1 / 2}}{|B \backslash \Omega|} \int_{B \backslash \Omega}|R-\nabla \varphi(x)| d x \text { for all } R \in \mathbb{O}_{+}^{n} .
$$

By combining these inequalities, we next deduce that

$$
\|\nabla \boldsymbol{\varphi}-\mathbf{I}\|_{L^{2}(B)} \leq C_{0}\left(\int_{B} \inf _{Q \in \mathbb{O}_{+}^{n}}|\nabla \varphi(x)-Q|^{2} d x\right)^{1 / 2}, \text { where } C_{0}=K^{1 / 2}\left(1+\frac{|B|^{1 / 2}}{|B \backslash \Omega|^{1 / 2}}\right) .
$$

Using that $\inf _{Q \in \mathbb{O}_{+}^{n}}|F-Q|^{2} \leq\left|F^{T} F-I\right|^{2}$ for all $F \in \mathbb{M}_{+}^{n}$, we finally obtain that

$$
\|\nabla \boldsymbol{\varphi}-\mathbf{I}\|_{L^{2}(\Omega)}=\|\nabla \boldsymbol{\varphi}-\mathbf{I}\|_{L^{2}(B)} \leq C_{0}\left\|\nabla \varphi^{T} \nabla \boldsymbol{\varphi}-\mathbf{I}\right\|_{L^{2}(B)}=C_{0}\left\|\nabla \boldsymbol{\varphi}^{T} \nabla \boldsymbol{\varphi}-\mathbf{I}\right\|_{L^{2}(\Omega)} .
$$

Now, by changing variables in the integrals defining the $L^{2}(\Omega)$-norms above, we obtain that

$$
\int_{\Omega}|\nabla \mathbf{u}-\nabla \mathbf{v}|^{2} \frac{\operatorname{det}(\nabla \mathbf{v})}{|\nabla \mathbf{v}|^{2}} d y \leq C_{0}^{2} \int_{\Omega}\left|\nabla \mathbf{u}^{T} \nabla \mathbf{u}-\nabla \mathbf{v}^{T} \nabla \mathbf{v}\right|^{2}\left|(\nabla \mathbf{v})^{-1}\right|^{4} \operatorname{det}(\nabla \mathbf{v}) d y
$$

Since $|\nabla \mathbf{v}(y)-I| \leq \lambda<1$ and $\left|\nabla \mathbf{v}^{-1}(y)-I\right| \leq \frac{\lambda}{1-\lambda}$ for all $y \in \bar{\Omega}$, we deduce that the singular values of the matrix $\nabla \mathbf{v}(y)$ are contained in the interval $\left[\frac{1-\lambda}{\lambda+(1-\lambda)|I|},|I|+\lambda\right]$. Therefore there exists constants $C_{1}>0$ and $C_{2}<\infty$ such that $\frac{\operatorname{det}(\nabla \mathbf{v})}{|\nabla \mathbf{v}|^{2}} \geq C_{1}(1-\lambda)^{n}$ and $\left|(\nabla \mathbf{v})^{-1}\right|^{4} \operatorname{det}(\nabla \mathbf{v}) \leq \frac{C_{2}}{(1-\lambda)^{4}}$. Combined with the previous inequality, this implies that

$$
\|\nabla \mathbf{u}-\nabla \mathbf{v}\|_{L^{2}(\Omega)} \leq \frac{C_{0}\left(C_{2} / C_{1}\right)^{1 / 2}}{(1-\lambda)^{2+n / 2}}\left\|\nabla \mathbf{u}^{T} \nabla \mathbf{u}-\nabla \mathbf{v}^{T} \nabla \mathbf{v}\right\|_{L^{2}(\Omega)} .
$$

\section{Proof of Theorem 2}

The assumptions on the function $W$ imply that $J(\mathbf{u}) \in \mathbb{R} \cup\{+\infty\}$ is well defined. They also imply that the Euler-Lagrange equation formally derived from the total energy $J: \mathcal{M} \rightarrow \mathbb{R} \cup\{+\infty\}$, that is

$$
-\operatorname{div}\left(\nabla \mathbf{v} \frac{\partial W}{\partial E}(\cdot, \mathbf{E}(\mathbf{v}))\right)=\mathbf{f} \text { in } \Omega \text { and } \mathbf{v}(x)=x \text { for all } x \in \Gamma,
$$

has a solution $\mathbf{v}=\mathbf{v}(\mathbf{f}) \in W^{2, p}\left(\Omega ; \mathbb{R}^{3}\right)$ given by the implicit function theorem for any $\mathbf{f} \in L^{p}\left(\Omega ; \mathbb{R}^{3}\right)$ such that $\|\mathbf{f}\|_{L^{p}(\Omega)}<\delta$; cf., e.g., Quintela-Estevez [7, Theorem 4.5]. Moreover, $\mathbf{v}$ is of class $\mathcal{C}^{1}$ and injective from $\bar{\Omega}$ into $\mathbb{R}^{3}$ (cf. Ciarlet [3, Theorems 5.5.1 and 5.5-2]) and

$$
\lambda(\delta):=\sup \left\{\|\nabla \mathbf{v}(\mathbf{f})-\mathbf{I}\|_{L^{\infty}(\Omega)} ; \mathbf{f} \in L^{p}\left(\Omega ; \mathbb{R}^{3}\right),\|\mathbf{f}\|_{L^{p}(\Omega)}<\delta\right\} \rightarrow 0 \text { as } \delta \rightarrow 0 .
$$

Choosing $\delta$ sufficiently small, we have $\|\mathbf{E}(\mathbf{v})\|_{L^{\infty}(\Omega)} \leq 3\|\nabla \mathbf{v}-\mathbf{I}\|_{L^{\infty}(\Omega)}<3 \lambda(\delta)<\varepsilon$. Then

$$
J(\mathbf{u})-J(\mathbf{v}) \geq \int_{\Omega}\left(\frac{\partial W}{\partial E}(\cdot, \mathbf{E}(\mathbf{v})):(\mathbf{E}(\mathbf{u})-\mathbf{E}(\mathbf{v}))+\alpha|\mathbf{E}(\mathbf{u})-\mathbf{E}(\mathbf{v})|^{2}\right) d x-\int_{\Omega} \mathbf{f} \cdot(\mathbf{u}-\mathbf{v}) d x .
$$


But

$$
\frac{\partial W}{\partial E}(\cdot, \mathbf{E}(\mathbf{v})):(\mathbf{E}(\mathbf{u})-\mathbf{E}(\mathbf{v}))=\frac{\partial W}{\partial E}(\cdot, \mathbf{E}(\mathbf{v})): \nabla \mathbf{v}^{T} \nabla(\mathbf{u}-\mathbf{v})+\frac{\partial W}{\partial E}(\cdot, \mathbf{E}(\mathbf{v})): \frac{\nabla(\mathbf{u}-\mathbf{v})^{T} \nabla(\mathbf{u}-\mathbf{v})}{2}
$$

(since $\frac{\partial W}{\partial E}(x, \mathbf{E}(\mathbf{v})(x)) \in \mathbb{S}^{3}$ for all $\left.x \in \bar{\Omega}\right)$ and

$$
\int_{\Omega} \frac{\partial W}{\partial E}(\cdot, \mathbf{E}(\mathbf{v})): \nabla \mathbf{v}^{T} \nabla(\mathbf{u}-\mathbf{v}) d x=-\int_{\Omega} \operatorname{div}\left(\nabla \mathbf{v} \frac{\partial W}{\partial E}(\cdot, \mathbf{E}(\mathbf{v}))\right) \cdot(\mathbf{u}-\mathbf{v}) d x .
$$

Hence

$$
\begin{aligned}
J(\mathbf{u})-J(\mathbf{v}) & \geq \int_{\Omega} \frac{\partial W}{\partial E}(\cdot, \mathbf{E}(\mathbf{v})): \frac{(\nabla \mathbf{u}-\nabla \mathbf{v})^{T}(\nabla \mathbf{u}-\nabla \mathbf{v})}{2} d x+\alpha \int_{\Omega}|\mathbf{E}(\mathbf{u})-\mathbf{E}(\mathbf{v})|^{2} d x \\
& \geq \frac{\alpha}{4}\left\|\nabla \mathbf{u}^{T} \nabla \mathbf{u}-\nabla \mathbf{v}^{T} \nabla \mathbf{v}\right\|_{L^{2}(\Omega)}^{2}-\frac{1}{2}\left\|\frac{\partial W}{\partial E}(\cdot, \mathbf{E}(\mathbf{v}))\right\|_{L^{\infty}(\Omega)}\|\nabla \mathbf{u}-\nabla \mathbf{v}\|_{L^{2}(\Omega)}^{2} .
\end{aligned}
$$

Then the nonlinear Korn inequality of Theorem 1 shows that, for some constant $C$,

$$
J(\mathbf{u})-J(\mathbf{v}) \geq\left(\frac{\alpha(1-\lambda(\delta))^{7}}{4 C^{2}}-\frac{1}{2}\left\|\frac{\partial W}{\partial E}(\cdot, \mathbf{E}(\mathbf{v}))\right\|_{L^{\infty}(\Omega)}\right)\|\nabla \mathbf{u}-\nabla \mathbf{v}\|_{L^{2}(\Omega)}^{2} .
$$

But $\lim _{\delta \rightarrow 0}\left(\frac{\alpha(1-\lambda(\delta))^{7}}{4 C^{2}}-\frac{1}{2}\left\|\frac{\partial W}{\partial E}(\cdot, \mathbf{E}(\mathbf{v}))\right\|_{L^{\infty}(\Omega)}\right)=\frac{\alpha}{4 C^{2}}$. Hence $J(\mathbf{u})-J(\mathbf{v})>0$ for all $\mathbf{u} \in \mathcal{M}, \mathbf{u} \neq \mathbf{v}$, if $\delta$ is chosen small enough.

\section{References}

[1] J. Ball, Convexity conditions and existence theorems in nonlinear elasticity, Arch. Rational Mech. Anal. 63, 337-403 (1977).

[2] D. Blanchard, Personal communication.

[3] P.G. Ciarlet, Mathematical Elasticity, Volume I: Three-Dimensional Elasticity, North-Holland, Amsterdam, 1988.

[4] P.G. Ciarlet and C. Mardare, Continuity of a deformation in $H^{1}$ as a function of its Cauchy-Green tensor in $L^{1}$, J. of Nonlinear Science 14, 415-427 (2004).

[5] P.G. Ciarlet and C. Mardare, Existence theorems in intrinsic nonlinear elasticity, J. Math. Pures Appl. 94, 229-243 (2010).

[6] G. Friesecke, R.D. James, and S. Müller, A theorem on geometric rigidity and the derivation of nonlinear plate theory from three dimensional elasticity, Comm. Pure Appl. Math. 55, 1461-1506 (2002).

[7] P. Quintela-Estevez, Critical points in the energy of hyperelastic materials, RAIRO Model. Math. Anal. Numer. 24, 103-132 (1990).

[8] K. Zhang, Energy minimizers in nonlinear elastostatics and the implicit function theorem, Arch. Rational Mech. Anal. 114, 95-117 (1991). 